\title{
MiNIREVIEW
}

\section{Leukocyte-Endothelial Cell Interactions in the Inflammatory Response}

\author{
William A. Muller \\ Department of Pathology and Graduate Program in Immunology, Weill Medical College of Cornell University, \\ New York, New York
}

\begin{abstract}
$I_{\text {to }}$ nflammation is the response of vascularized tissue o damage. It is a complex response set in motion in any tissue by virtually any injury due to any cause. It is an attempt to restore homeostasis. In the early history of Homo sapiens, when lifespans were relatively short and our ancestors were likely to die from infectious diseases and trauma, the ability to fight off pathogens and heal wounds was even more critical for the survival of the species than it is today. The inflammatory response is still critical for these purposes. However, now that we are living longer, we have come to realize that inflammation is a doubleedged sword. The same inflammatory processes that are so effective at clearing pathogens and repairing damaged skin are the cause of most human pathology today. Atherosclerosis, autoimmune diseases, pulmonary fibrosis, asthma, and many other common human afflictions are all conditions that result when the inflammatory response is uncontrolled, self-directed, or in the wrong place at the wrong time. These conditions generally affect humans past the traditional breeding age and thus these untoward aspects of inflammation were not selected out during evolution. As a result, there is now far more effort to develop potent anti-inflammatory therapies than there is to develop treatments to boost the inflammatory response.

What is needed is the therapeutic equivalent of "smart bombs"-the ability to selectively inhibit inflammation where it is damaging without interfering with beneficial functions of the inflammatory response. To design rational therapies, a more thorough understanding of the cellular and molecular basis of inflammation is necessary. A great deal of research
\end{abstract}

\section{Received February 1, 2002}

The author is supported by grants from the National Institutes of Health, HL46849 and HL64774.

Address reprint requests to: Dr. William A. Muller, Professor, Department of Pathology and Graduate Program in Immunology, Weill Medical College of Cornell University, 1300 York Avenue, New York, NY 10021. E-mail: wamuller@med.cornell.edu has been carried out in this area over the past two decades and significant progress has been made. Along with this progress has come an array of jargon and hundreds of molecules denoted by cluster of differentiation (CD) numbers that make following the field daunting for those not involved in it (see Table 1) This brief overview is intended to introduce the nonspecialist to the general concepts in the field and the important areas for future research. A general paradigm of leukocyte-endothelial cell interactions in inflammation is presented that is generally agreed on in the field. This is obviously oversimplified; alternative interactions do exist. In particular, there are certain adhesion molecules that are critical to inflammation in some tissues but not in others. The reader is referred to more detailed reviews cited in each section for a more thorough discussion.

\section{The Site of Inflammation: An Overview}

Inflammation is a stereotyped series of responses involving soluble factors, vascular permeability, and leukocyte migration. Soluble mediators such as histamine released from tissue mast cells or kinins activated from circulating precursor molecules by proteolysis trigger the immediate and transient vascular response to tissue damage. These changes in local blood flow and vascular permeability produce the classic signs of inflammation, tumor, rubor, calor, dolor, described by Celsus two thousand years ago. The vascular changes set the stage for the definitive phases of inflammation when circulating leukocytes exit the circulation at the site of damage to destroy pathogens, clean up cell debris, and begin the process of tissue repair.

The transient endothelial contraction that leads to vascular leakage takes place at postcapillary venules. These vascular changes are themselves deserving of a separate review. This report focuses on the cellular events of inflammation. For our purposes, the increased permeability leads to hemoconcentration that slows blood flow locally. As blood flow shear rate 
Table 1. Common Leukocyte-Endothelial Cell Adhesion Molecules in Inflammation

\begin{tabular}{|c|c|c|c|c|c|}
\hline Leukocyte Molecule & $\begin{array}{l}C D^{a} \text { and Integrin } \\
\text { Nomenclature }\end{array}$ & $\begin{array}{l}\text { Leukocytes } \\
\text { Expressing }^{b}\end{array}$ & Actions & $\begin{array}{l}\text { Endothelial Cell } \\
\text { Counter-Ligand }\end{array}$ & $\begin{array}{l}\text { CD Nomenclature of } \\
\text { Counter-Ligand }\end{array}$ \\
\hline L-selectin & CD62L & PMN, Mo, T, B, NK & Tethering, rolling & $\begin{array}{l}\text { Sialyl-Lewis }{ }^{x} \text { on } \\
\text { appropriate ligand }\end{array}$ & CD34 others \\
\hline PSGL-1 & CD162 & PMN, Mo, T, B, NK & Tethering, rolling & P-selectin & CD62P \\
\hline $\begin{array}{l}\text { ESL-1 and CLA } \\
\text { bearing sialyl } \\
\text { Lewis }^{x}\end{array}$ & CD15s & PMN, Mo, T, B, NK & Tethering, rolling & E-selectin & CD62E \\
\hline VLA-4 & CD49d/CD29 $\left(\alpha_{4} \beta_{1}\right)$ & $\begin{array}{c}\text { Mo, B, Eo }>\text { NK, T, } \\
(\mathrm{PMN})^{d}\end{array}$ & $\begin{array}{l}\text { Tethering, rolling } \\
\text { Tight adhesion }\end{array}$ & VCAM-1 & CD106 \\
\hline LFA-1 & $\mathrm{CD11a/CD18}\left(\alpha_{\mathrm{L}} \beta_{2}\right)$ & PMN, Mo, T, B, NK & Tight adhesion & $\begin{array}{l}\text { ICAM-1 } \\
\text { ICAM-2 }\end{array}$ & $\begin{array}{l}\text { CD54 } \\
\text { CD102 }\end{array}$ \\
\hline Mac-1, CR3 & $\mathrm{CD} 11 \mathrm{~b} / \mathrm{CD} 18\left(\alpha_{\mathrm{M}} \beta_{2}\right)$ & PMN, Mo, NK & Tight adhesion & ICAM-1 & CD54 \\
\hline PECAM-1 & CD31 & $\begin{array}{c}\text { PMN, Mo, NK, subsets } \\
\text { of T cells }\end{array}$ & Diapedesis & PECAM-1 & CD31 \\
\hline CD99 & CD99 & Mo, PMN, T, B, NK & Diapedesis & CD99 & CD99 \\
\hline
\end{tabular}

PSGL-1, P-selectin glycoprotein 1; VLA-4, very late antigen 4; VCAM-1, vascular cell adhesion molecule 1; LFA-1, leukocyte function antigen-1; ICAM-1, -2, intercellular adhesion molecule-1, -2; CR3, complement receptor 3; PECAM-1, platelet/endothelial cell adhesion molecule-1. Adapted from (Hajjar et al, 2001) with permission from the publisher.

${ }^{a} \mathrm{CD}$, Cluster of differentiation, the number assigned to the antigen by the International Workshops on Leukocyte Typing, as defined by monoclonal antibody recognition.

${ }^{b}$ PMN, neutrophils; Mo, monocytes; T, T lymphocytes; B, B lymphocytes; NK, natural killer cells; Eo, eosinophils.

${ }^{c}$ ESL-1, E-selectin ligand, a protein with homology to fibroblast growth factor, has been identified in mice. CLA, cutaneous lymphocyte antigen, a molecule on the surface of skin-homing T cells related to PSGL-1, directs them to skin venules via interactions with E-selectin. The CD number refers to the sialyl Lewis ${ }^{\times}$carbohydrate moiety no matter what protein backbone it is expressed on.

${ }^{d}$ (PMN) Although PMN were not believed to express VLA-4, recent evidence suggests that this integrin may be expressed on or be induced on PMN under certain conditions.

drops below a critical value, leukocytes, which are normally swept along in the central stream of laminar blood flow, are afforded access to the endothelium lining the postcapillary venules. This, in turn, allows the leukocyte-endothelial cell interactions that promote leukocyte extravasation. In most tissues and most inflammatory settings, leukocytes leave the bloodstream at the level of postcapillary venules (Marchesi, 1961; Marchesi and Florey, 1960).

Intravital microscopy studies show that leukocytes attaching to the blood vessel wall leave the circulation and enter the tissues within a minute or two. However, this rapid process entails a coordinated series of different adhesion events, which must occur in the precise order for the process to succeed-like a combination lock in which the proper signals must be given in the correct order. Because the inflammatory response can harm the host tissues if uncontrolled or misdirected, one can imagine that this is a safeguard to prevent unwanted damage due to inflammation in case leukocytes or endothelial cells become accidentally activated.

Although there are exceptions, a general paradigm for this process has each successive step governed by a different class of adhesion molecules on the leukocytes or endothelium (see Fig. 1). The initial loose interactions of "rolling" are mediated by the selectin family of adhesion molecules and their carbohydrate ligands on the apposing cell. Rolling brings leukocytes into close enough contact with the endothelium that receptors on their surfaces can be engaged by molecules presented on the endothelial cells to activate the next step: firm adhesion. This step involves members of the integrin family of adhesion molecules on leukocytes binding to their counter-receptors on endothelial cells. As far as the endothelial counter-receptors have been identified, they are members of yet another adhesion molecule family, the immunoglobulin gene superfamily. When leukocytes actually squeeze between the apposing endothelial cells (a process referred to as diapedesis or transmigration) the rules change a bit. Where specific adhesion molecules that govern this process have been identified, adhesion is mediated mostly by homophilic interactions between the same molecules expressed on both leukocytes and endothelial cells. Diapedesis and the subsequent migration of leukocytes through interstitial tissue are believed to be stimulated by gradients of soluble or surface-bound chemoattractants from the inflammatory environment including bacterial cell products, factors released from necrotic host cells, and chemokines made by host cells in response to inflammatory stimuli. The need for a gradient of chemoattractant to stimulate diapedesis has been challenged recently (Cinamon et al, 2001). We can dissect leukocyte emigration into these discrete steps because we have reagents that block the process at each step. There may well be steps intermediate or distal to the known ones governed by yet undiscovered molecular interactions (Muller, 1999; Muller et al, 1993; Schenkel et al, 2002).

In this review, we will use the term "leukocytes" to refer to all circulating white blood cells. In some cases, specific molecular events have been demonstrated only for subsets of leukocytes such as neutrophils, monocytes, or T lymphocytes. In general, however, 
Capture/Tethering

Rolling

Activation

Adhesion

Diapedesis

Traversing

Basal Lamina

Migration through ECM

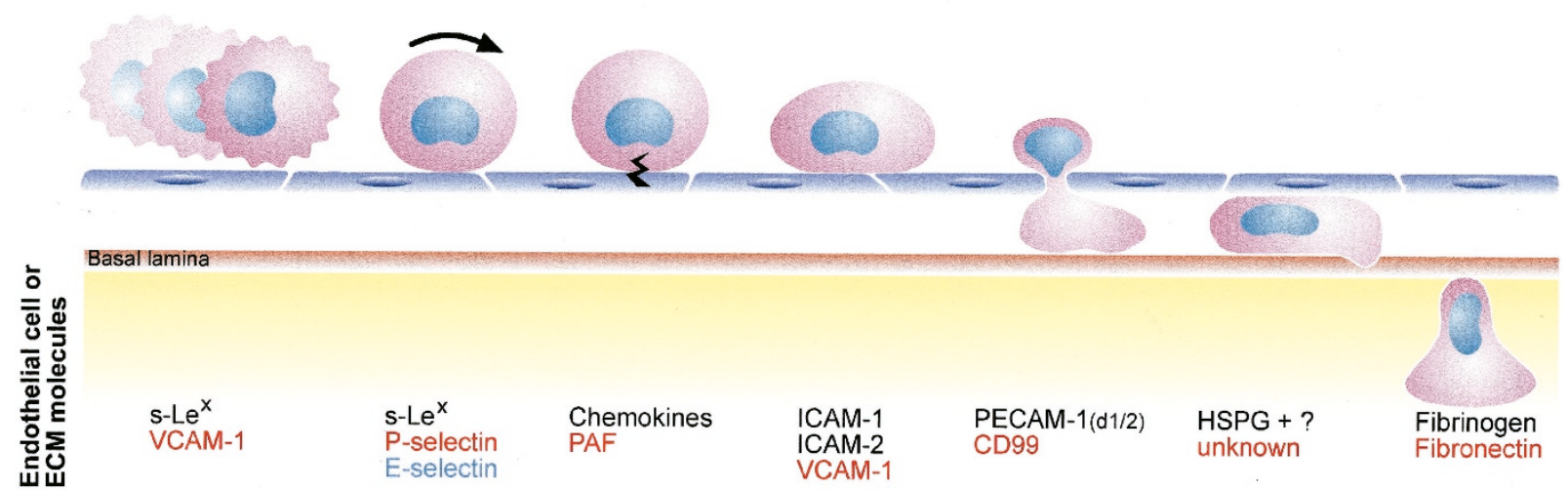

Figure 1.

Sequential steps in leukocyte emigration are controlled by specific adhesion molecules on leukocytes and endothelial cells. The various steps of leukocyte emigration described in the text are depicted schematically here. For each step the interacting pairs of adhesion molecules, ligands, or counter-receptors expressed by the leukocytes and endothelial cells or extracellular matrix are shown in the same color. This diagram is not inclusive, and other molecules may mediate each of these events for distinct leukocyte types under different inflammatory conditions. For the Capture/Tethering step, the protrusions on the leukocyte surface are meant to represent the microvilli that bear L-selectin or VLA-4. The lightning bolt at the Activation step represents the triggering of inside-out activation of leukocyte integrins by signals from the endothelium and endothelial surface via $G$ protein coupled receptors. The basal lamina (orange strip) is depicted as separate from the remainder of the extracellular matrix (ECM), because migration across the subendothelial basal lamina seems to be a separate step controlled by distinct molecules (Liao et al, 1995; Wakelin et al, 1996). However, these steps may not be molecularly distinct (see text). Because the exact $\beta_{1}$ integrin(s) that are involved in migration through ECM via fibronectin interactions have not been defined, the leukocyte molecules are designated simply as $\beta_{1}$. ESL, E-selectin ligand; HSPG, heparan sulfate proteoglycan; ICAM, intercellular adhesion molecule; LFA, leukocyte function associated antigen; PAF, platelet activating factor; PAF-R, PAF receptor; PECAM-1, platelet/endothelial cell adhesion molecule-1; PECAM-1 (d1/2), interaction involves immunoglobulin domains 1 and/or 2 of PECAM-1; PECAM-1 (d6), interaction involves immunoglobulin domain 6 of PECAM-1; PSGL, P-selectin glycoprotein ligand; s-Le , sialyl-Lewis ${ }^{x}$ carbohydrate antigen; VLA, very late antigen.

where leukocyte subsets have been compared, the molecular interactions governing teukocyteendothelial cell interactions are similar. The degree to which distinct steps in the emigration process are reliant on a specific adhesion molecule may vary among cell types due to the expression on some cells of alternative molecules that can carry out that particular step. In other words, although the process of emigration is similar for all types of leukocytes, the exact adhesion molecules that they use to carry out the rolling, adhesion, and transmigration steps may vary among the leukocyte types. In addition, different adhesion molecules may predominate in different tissues or under different inflammatory conditions. Finally, there is a great deal of redundancy in the control of this process. Several different molecules can carry out the same step of leukocyte emigration. This has made analysis of the process difficult, because inhibiting the function of a molecule that really plays a role in leukocyte emigration may not lead to an observable phenotype if other molecules take over for it.

\section{Tethering and Rolling}

Because leukocytes cannot swim, they must first attach to the endothelium lining the blood vessel wall before they can pass through into the inflamed tissues. The hemoconcentration that follows vascular leakage in the early phase of the inflammatory response slows blood flow sufficiently so that leukocytes can make functional contacts with the endothelium. These initial contacts serve to attach or "tether" the white cells to the endothelium. These initial capturing interactions are generally mediated by the selectin family of adhesion molecules and their sulfated, sialylated, fucosylated, glycoprotein ligands (Carlos and Harlan, 1994; Etzioni et al, 1999; Vestweber and Blanks, 1999). These generally are of the configuration of the blood group carbohydrate sialyl-Lewis ${ }^{x}$. For optimal P-selectin and L-selectin binding, tyrosine residues on the amino terminus of the protein bearing sialyl-Lewis ${ }^{\times}$must be sulfated (Pouyani and Seed, 1995; Sako et al, 1995; Vestweber and Blanks, 1999). Biosynthesis of selectin ligands and their specific recognition by selectins is a complex issue, which is beyond the scope of this brief overview. The reader is referred to the preceding reviews (Carlos and Harlan, 1994; Etzioni et al, 1999; Vestweber and Blanks, 1999). Recent data show that the integrin $\alpha_{4} \beta_{1}$ can mediate also rolling of T cells (Berlin et al, 1995) and neutrophils (Henderson et al, 2001).

The selectin family consists of three related molecules. Each has an amino terminal lectin domain, which is primarily responsible for its adhesive activity. This is followed by an epidermal growth factor (EGF) motif and a variable number of repeated complement 
regulatory domains, (so named due to their similarity to those in complement regulatory proteins) before a transmembrane domain and cytoplasmic tail. The major structural differences between the selectins are due to the number of repeated complement regulatory domains. L-selectin, expressed by leukocytes, has two. E-selectin, whose expression is limited to endothelial cells has six of these domains. The human form of P-selectin, made by platelets and endothelium has nine complement protein-related domains. The role of these domains in selectin function is not known, although in some model systems, creating recombinant selectins with this portion of the molecule truncated resulted in altered binding activity (Vestweber and Blanks, 1999).

Because histamine is released by mast cells early in inflammation and is responsible for the early vascular leakage, and because histamine triggers the rapid exocytosis of $\mathrm{P}$-selectin from endothelial cells, one might expect that rolling would begin early in inflammation during this histamine-dependent stage. Studies by Kubes and Kanwar (1994) showed that histamine release from mast cells was indeed responsible for the "baseline rolling" seen in intravital microscopy experiments. Degranulation of mast cells is due to the trauma that takes place during the surgical preparation of mice and rats for these studies. When prevented by preadministration of a mast cell granule stabilizer, baseline rolling was markedly reduced (Kubes and Kanwar, 1994).

L-selectin on leukocytes has been shown to be enriched on microvillous projections of the cell membrane, whereas the integrin molecules that mediate the next step of emigration (see below) are restricted to the body of the leukocyte between microvilli (von Adrian et al, 1995). In this way, the molecules mediating the initial tethering of leukocytes contact the endothelium first. Soon after this initial contact, the microvilli retract, allowing contact of the integrins with their counter-receptors on the endothelium. Recent data demonstrate that the integrin $\alpha_{4} \beta_{1}$ on leukocytes interacting with vascular cell adhesion molecule-1 (VCAM-1) on inflamed endothelium may be able to play a similar role (Henderson et al, 2001). In such cases, $\alpha_{4} \beta_{1}$ has been shown to be enriched in microvillous projections, similar to L-selectin (Berlin et al, 1995).

One of the safeguards preventing unwanted inflammation is the regulated expression of cell adhesion molecules and/or their ligands. For example, E-selectin is not expressed by resting endothelium. Its expression is induced after activation of the endothelium by inflammatory cytokines and other stimuli and involves de novo gene transcription. P-selectin is stored preformed in the $\alpha$ granules of platelets and within the Weibel-Palade bodies of endothelial cells. It can be rapidly brought to the surface by degranulation of these compartments, in response to thrombin or histamine, respectively. Under conditions of chronic inflammatory stimulation, P-selectin can be stably expressed on the cell surface (Pan et al, 1998; Yao et al, 1996). On the other hand, leukocytes circulate constitutively expressing L-selectin, but its glycoprotein ligands must be properly decorated with the correct carbohydrate residues to promote interaction. One such ligand, CD34, is a glycoprotein expressed on the surfaces of most endothelial cells. On stimulation by inflammatory mediators, endothelial cell fucosyl transferases and other appropriate enzymes are induced and begin adding the proper sugar residues to the protein backbones of CD34 and other selectin ligand scaffolds. The major leukocyte receptor for the endothelial cell selectins, P-selectin glycoprotein ligand-1 (PSGL-1), is constitutively expressed on neutrophils and $\mathrm{T}$ cells, although its carbohydrate residues may require modification for optimal binding. Thus, the different expression patterns of the three selectins (constitutive $=\mathrm{L}$; hyperacute $=\mathrm{P}$; acute $=\mathrm{E}$; chronic $=\mathrm{P}$ ) ensure that there are molecules to support leukocyte rolling throughout the time course of inflammation.

The importance of fucose residues in the selectin ligands is underscored by a congenital defect known as leukocyte adhesion deficiency type II (LAD II). This is a rare (four patients reported; Etzioni et al, 1999) defect in the enzyme that converts mannose to fucose. As a result, selectin ligands are severely underfucosylated (approximately $2 \%$ of normal levels) and there is marked deficiency in the ability of leukocytes from these patients to roll on endothelium (Etzioni et al, 1999). Clinically, however, this condition does not produce the life-threatening infections seen with LAD type I (see below), probably because $\alpha_{4} \beta_{1}$ and perhaps other molecules can take over the rolling function for the deficient leukocytes.

\section{Arrest and Adhesion}

The processes of tethering and rolling are reversible. Many leukocytes that roll in vivo will not stop, but dissociate from the vessel surface and reenter the bloodstream. To stop rolling, the low affinity transient interactions of rolling must be replaced by high affinity adhesion between the leukocyte and the endothelial surface. Such high affinity interactions are triggered by stimuli presented to the leukocyte on the endothelial surface. These may be intrinsic endothelial cell surface molecules (Muller, 1999) or molecules such as chemokines from the inflammatory site that are bound to endothelial surface glycosaminoglycans and presented to the leukocytes (Tanaka et al, 1993).

Chemokines, a related group of low molecular weight (8-14 kd) heparin-binding "chemotactic cytokines," are secreted by a variety of cells in the body under inflammatory and noninflammatory conditions. Chemokines are divided into four families depending upon the location of intrachain disulfide bonds. They attract leukocytes via specific heptahelical, pertussis toxin-sensitive, G protein-coupled receptors. Specific chemokines tend to be more potent for certain subsets of leukocytes than for others. For example, IL-8 is most potent for neutrophils, MCP-1 for monocytes, and SDF-1 for T cells. The reader is referred to any of a large number of reviews on chemokines and their 
biology (Baggiolini, 1998, 2001; Campbell and Butcher, 2000; Zlotnick and Yoshie, 2000). Although chemokines have received a great deal of attention recently for their role in attracting leukocytes and activating adhesion, they are by no means the only agonists that can do this. The common denominator is that interaction with these molecules activates the leukocyte, resulting in adhesion by yet another family of adhesion molecules, the integrins.

Integrins are heterodimeric receptors consisting of paired $\alpha$ and $\beta$ chains. Most integrin families (defined by the $\beta$ chain) bind cells to extracellular matrix. Leukocytes possess a unique family of integrins, the $\beta_{2}$ or leukocyte-specific integrins (also known as CD18), that are primarily involved in their adhesion to other cells. Of the four identified $\alpha$ chains that pair with CD18, $\alpha_{\mathrm{L}}$ (CD11a) and $\alpha_{\mathrm{M}}$ (CD11b) are the most important for the adhesion of leukocytes to endothelium. The integrins formed, $\alpha_{\mathrm{L}} \beta_{2}$ (also known as CD11a/CD18 and leukocyte function associated antigen 1, LFA-1) and $\alpha_{\mathrm{M}} \beta_{2}$ (also known as CD11b/CD18 and Mac-1) are expressed to different extents on the various leukocyte types. In general, they can substitute for each other, and to effectively inhibit leukocyte adhesion therapeutically in most models, both integrins (or the common $\beta$ chain) must be blocked. In addition many leukocytes express the $\beta_{1}$ integrin $\alpha_{4} \beta_{1}$ (very late antigen $4, \mathrm{VLA}-4)$. This can adhere to a molecule expressed on the surface of inflamed endothelium that is distinct from the counter-receptor for the $\beta_{2}$ integrins (see below.)

Integrins exist in a low and high affinity binding state with the low affinity state favored. They must be activated to favor the high affinity state and efficiently bind their ligands. This can be done in two ways. Avidity modulation clusters the integrins in the plane of the membrane. Making the molecular density greater increases the probability that a molecule in that cluster will be in the active conformation at any given time. Affinity modulation involves a conformational change in the integrin chains that favors their assumption of the active state. Signals that trigger affinity modulation can be transduced by a variety of receptors for inflammatory mediators. Recent research has highlighted the role of heptahelical receptors for chemokines and formylated peptides, all coupled to heterotrimeric G-proteins, in the transmission of signals that activate leukocyte integrins.

In a typical scenario, the neutrophil activator and chemoattractant interleukin 8 (IL-8) may be made by macrophages at a site of inflammation and transcytosed to the apical surface of the overlying endothelium (Middleton et al, 1997). Alternatively or additionally, activated endothelial cells at a site of inflammation may produce IL-8 themselves (Kaplanski et al, 1994, 1997). IL-8, like other chemokines, is a heparin binding protein and binds to glycosaminoglycans on the endothelial surface. It is thus presented to the rolling leukocytes. When a rolling leukocyte binds interleukin 8 (IL-8, also known by the chemokine nomenclature CXCL8) via its receptor (CXCR1 or CXCR2), the response triggered in the leukocyte be- gins a signaling cascade within the leukocyte that activates the leukocyte integrin via its cytoplasmic tail. This phenomenon is thus called "inside-out" signaling, because the integrin is activated by signals from the inside resulting in enhanced adhesion on the outside (Hynes, 1992). Molecules other than chemokines can trigger leukocyte adhesion. Platelet activating factor (PAF) on the endothelial surface via its receptor on leukocytes (Zimmerman et al, 1990) as well as crosslinking L-selectin on leukocytes (Simon et al, 1995) can trigger inside-out integrin activation.

When activated, the leukocyte integrins bind to their ligands or counter-receptors on endothelium. These molecules, intercellular adhesion molecule (ICAM)-1 and -2 as well as vascular cell adhesion molecule-1 (VCAM-1) make high affinity adhesions with the leukocyte integrins, allowing them to stop rolling and begin crawling on the endothelial cell surface-the next step in their migration. The avidity of the counterreceptors can be regulated as well. The active form of ICAM-1 functions as a dimer (Miller et al, 1995; Reilly et al, 1995).

Adhesion is another regulated step in inflammation. ICAM-1 and VCAM-1 are expressed at low levels on endothelial cells of most vascular beds in the absence of inflammation. Their expression is dramatically increased in response to inflammatory cytokines (Pober and Cotran, 1990). Experiments using human umbilical vein endothelial cells in vitro show that this upregulation can reach 50- to 100-fold (Muller and Weigl, 1992). Cytokine-induced increase in expression of ICAM- 1 and VCAM- 1 can be seen within hours of stimulation, but levels peak on a time scale of days (Carlos and Harlan, 1994; Pober and Cotran, 1990).

Many studies in vitro and in vivo using monoclonal antibodies to block the function of leukocyte integrins (Carlos and Harlan, 1994) and studies of mice in which one or more of the integrins were genetically deleted (Etzioni et al, 1999) demonstrate that these molecules play a critical role in leukocyte adhesion. Additional compelling evidence that the $\beta_{2}$ integrins are critical for neutrophils in the inflammatory response comes from studies of an inherited defect known as leukocyte adhesion deficiency type I (LAD I) (Anderson and Springer, 1987; Etzioni et al, 1999). Patients with LAD I do not express significant levels of $\beta_{2}$ integrins on the surfaces of their leukocytes. This is due to an inherent defect in the common $\beta$ chain, CD18. Several genotypes have been described that encode mutations in CD18 that preclude its synthesis or render it nonfunctional. An incomplete $\beta$ chain is degraded in the Golgi apparatus and the corresponding $\alpha$ chains, which rely on pairing with the $\beta$ chain to get to the cell surface, are not expressed. The defects have incomplete penetrance. Severely affected individuals have undetectable surface levels of $\beta_{2}$ integrins. These individuals have a profound neutrophilia, but the neutrophils cannot bind to the endothelial cells and therefore cannot enter sites of inflammation. Typically, they die with bacterial overgrowth in their tissues in the absence of any tissue neutrophils. 
Some additional important features of the inflammatory response have been learned from the study of LAD I patients. First, those patients with a moderate phenotype have circulating leukocytes bearing only $2 \%$ to $5 \%$ of normal $\beta_{2}$ integrin levels. Yet their ability to handle infections is much closer to that of unaffected individuals. Therefore, approximately $5 \%$ of normal surface levels, when working properly, are sufficient to keep most people healthy. Second, LAD I patients do not show evidence of a defect in monocyte or lymphocyte function. These cells bear $\alpha_{4} \beta_{1}$ and other cell adhesion molecules that can take over for $\beta_{2}$ integrins. Finally, although human PMN seem to be primarily dependent on $\beta_{2}$ integrins for their adhesion to endothelium, in mice (Henderson et al, 2001) and rats (Issekutz et al, 1996) there is increasing evidence for a small, but important, pool of $\alpha_{4} \beta_{1}$ on the neutrophil surface that can take over for $\beta_{2}$ when the latter is blocked in experimental models.

\section{Migration on the Endothelial Surface}

Although the leukocyte may roll for several seconds over many endothelial cells before finally arresting, once tightly adherent to the endothelial cell surface, the leukocyte seems to migrate into the tissues by crossing the border of the endothelial cell it is adherent to. Migration on the surface of endothelium toward the junction requires leukocytes to maintain adhesion at the front end of the cell while letting go at the rear. Little is known about the regulation of these cycles. However, the distance traveled is not likely to be too great. Some studies show that most neutrophils arrest within one cell diameter of the junction they will migrate across (Gopalan et al, 2000).

Leukocyte migration on the apical surface of endothelium seems to be mediated by interactions between $\beta_{2}$ integrins of the leukocyte and ICAM molecules on the endothelial surface. Cell migration requires that leukocytes polarize with cycles of adhesion at the front and de-adhesion at the rear. Membrane protrusion/extension at the leading edge provides a mechanism to move the cell forward. At the same time, membrane is removed from the trailing edge and reincorporated into the main cell body. There is evidence that at least part of cell movement involves internalization of integrin-bearing membrane at the rear by endocytosis and recycling of that membrane to the front of the cell (Lawson and Maxfield, 1995). Although these studies were performed on cells migrating on vitronectin on coated glass, not on endothelial cells, a recent report on monocyte transmigration across endothelial cells supports the generalization of this idea. In that study, transmigration was blocked by inhibition of the small GTPase RhoA. The investigators found that $\beta_{2}$ integrins remained localized in the unretracted tail of the monocyte (Worthylake et al, 2001).

Once leukocytes arrest tightly on the endothelial surface and begin to transmigrate, it is generally believed that the subsequent process of transendothelial migration is independent of fluid shear (Smith et al, 1988). However, some recent papers provide data demonstrating more efficient adhesion (Cuvelier and Patel, 2001; Kitayama et al, 2000) or transmigration (Cinamon et al, 2001) under conditions of fluid shear. Although monocytes and neutrophils can transmigrate efficiently in the absence of flow in many in vitro models (Muller and Weigl, 1992; Shaw et al, 2001), T cell transmigration was remarkably more efficient in the presence of shear stress (Cinamon et al, 2001).

\section{Endothelial Junctions in Inflammation}

There are several well-documented reports of leukocytes migrating into sites of inflammation by passing through endothelial cells (Feng et al, 1998). However, most investigators agree that most leukocytes enter the sites of inflammation by passing between endothelial cells at their borders (reviewed in [Muller, 2001]). Endothelial cells are connected to each other by specific junctional structures at their borders (Dejana et al, 1995). These junctions must presumably be breached by transmigrating leukocytes. On the other hand, vascular integrity must be maintained during inflammation. Indeed, using in vitro models, considerable leukocyte traffic across endothelial cell monolayers can occur without an increase in the permeability or decrease in the electrical resistance of the monolayers (Huang et al, 1988, 1993). Experiments in which transendothelial migration led to a significant reduction in monolayer integrity or destruction of junctional components used high ratios of leukocytes to endothelial cells ( $\geq 10: 1$ ) (Allport et al, 1997; Del Maschio et al, 1996; Huang et al, 1993; Moll et al, 1998).

Adherens junctions and tight junctions encircle endothelial cells where they adhere to adjacent endothelial cells in the monolayers. Junctional adhesion molecules such as the vascular endothelial cadherin (VEcadherin) hold apposing endothelial cells together along these belts, with their cytoplasmic tails organizing actin-based cytoskeletal supports (Dejana et al, 1995). Tight junctions provide a true barrier to even small molecule movement across the junctions. Truly tight junctions are rare in endothelium, limited to cells comprising the blood-brain and blood-testis barriers. However, even the relatively leaky postcapillary venular endothelium has rudimentary tight junctions, which would serve as a barrier to leukocyte passage.

Some studies in vitro and in vivo suggest that neutrophils are more likely to migrate through a junction where three endothelial cells meet (tricellular junction) rather than a border between two endothelial cells (Burns et al, 1997, 2000). Studying leukocyte migration in various species, Burns and colleagues have found that approximately $50 \%$ to $75 \%$ of neutrophil transmigration takes place at tricellular borders. This implies that transmigration is highly selective for tricellular junctions, because they make up less than $10 \%$ of the endothelial border area. This preference for migration at tricellular borders may be spe- 
cific for neutrophils or for particular vascular beds. In a similar in vitro flow model, monocytes preferentially migrate across borders made by two endothelial cells (Shaw et al, 2001).

Recent data suggest mechanisms by which monolayer integrity could be maintained in the face of rates of transmigration that might be attained during inflammation. Adherens junctions seem to disorganize during transmigration of monocytes (Allport et al, 2000). VE-cadherin and the catenin molecules associated with it transiently disappear from the endothelial membrane at the border surrounding the migrating leukocyte. Yet, the entire junctional complex re-establishes itself within 5 minutes of monocyte migration (Allport et al, 2000). Tight junctions on venular endothelium are discontinuous, especially at tricellular borders where transmigration of neutrophils is particularly favored (Burns et al, 1997, 2000). In some cases these discontinuities may be large enough (1-2 $\mu \mathrm{m})$ to permit neutrophil passage. In other cases, retraction or "sliding" of tight junctional complexes, signaled perhaps by the increases in intracellular free calcium triggered by leukocyte adhesion (see below), may facilitate passage of the leukocytes without wholesale disruption of the junctions (Burns et al, 2001).

What is known about the inter- and intramolecular signaling that regulates diapedesis? Transmigration requires a transient increase in intracellular free calcium within the endothelial cells (Huang et al, 1993). If this intracellular free calcium rise is buffered, neutrophils can still adhere, but cannot transmigrate endothelial monolayers (Huang et al, 1993). The intracellular free calcium rise is restricted to endothelial cells that were adjacent to a transmigrating leukocyte (Su et al, 2000). The increase in intracellular free calcium activates myosin light chain kinase (MLCK), which may lead to endothelial cell retraction facilitating leukocyte passage (Hixenbaugh et al, 1997). In support of this, the MLCK inhibitor ML-9 blocked transendothelial migration and the accompanying actin cytoskeletal changes in an in vitro model (Saito et al, 1998).

There is evidence that soluble product(s) from leukocytes can trigger this calcium rise. Cationic protein(s) released by stimulated neutrophils can trigger a rise in endothelial cytosolic free calcium concentration similar to that triggered by migrating neutrophils themselves (Gautam et al, 2000). Recently, the heparinbinding protein azurocidin (also known as CAP37 or HBP) has been identified as one soluble product released from neutrophils that can trigger the rise in intracellular free calcium (Gautam et al, 2001). Soluble products of neutrophils may not be the only way of signaling the endothelial cells to permit transmigration. There is also a report that cross-linking E- or P-selectin or VCAM-1 on activated endothelial cells can trigger this calcium rise (Lorenzon et al, 1998). This intriguing work awaits confirmation by other groups, but suggests a way in which the calcium flux could be exquisitely localized to the endothelial cells surrounding the migrating leukocyte (Su et al, 2000).

\section{Diapedesis (Transendothelial Migration)}

Although the preceding steps in leukocyte emigration are all potentially reversible (that is, rolling cells may not adhere; adherent cells may detach and reenter the bloodstream), diapedesis is the "point of no return." Once a leukocyte commits itself to migrating across the endothelium, it does so, and its fate is determined. During diapedesis the leukocyte squeezes in ameboid fashion between the tightly apposed endothelial cells. This is a truly remarkable process. On a time scale of seconds the leukocyte undergoes a dramatic change in shape, extensively remodeling its cytoskeleton, and extending itself via a pseudopod across the endothelial border. Leukocytes, especially neutrophils, must be activated enough to adhere and carry out these functions, but not activated so much that they are stimulated to undergo an oxidative burst.

Diapedesis changes the subsequent physiology of the leukocytes. Neutrophils, which normally circulate for only a few hours before being removed by the spleen, receive antiapoptotic stimuli from inflamed endothelial cells that allows them to function at the site of inflammation (Coxon et al, 1999). Monocytes are stimulated to differentiate into either macrophages or dendritic cells (Randolph et al, 1998, 1999), preparing them for new roles as effector cells or antigenpresenting cells, respectively.

Unique molecular mechanisms govern this step in leukocyte migration. Whereas tethering, rolling, adhesion, and crawling on the apical surface all involve heterophilic interactions between one class of molecule on the leukocyte and another class of molecule on the endothelial cell, the two major known molecular interactions in diapedesis involve homophilic interaction between a molecule on the leukocyte and the same molecule on the endothelium at the junctions. These molecules, platelet/endothelial cell adhesion molecule-1 (PECAM-1/CD31) and CD99, act at sequential steps as the leukocyte crosses the endothelial barrier.

There are many reports that interfering with the function of $\beta_{2}$ integrins or VLA-4 on leukocytes, or their counter-receptors on endothelial cells, inhibit transmigration. This is certainly true. However, in the author's opinion, the inhibition of transmigration seen in these cases was due to the fact that these molecules mediate firm adhesion, the necessary prerequisite for transmigration. Blocking adhesion necessarily blocks transmigration (Muller, 1999). In an assay that distinguishes the role for a molecule in adhesion versus transmigration, leukocyte integrins were found to have no specific role in diapedesis, only in adhesion to the apical surface (Muller et al, 1993; Muller and Weigl, 1992). On the other hand, PECAM-1 and CD99 have no effect on adhesion of monocytes to the apical surface of endothelial cells, but act selectively in the borders between endothelial cells at the diapedesis step.

PECAM-1 is a member of the immunoglobulin gene (Ig) superfamily that is expressed diffusely on the surfaces of most leukocytes and is concentrated at 
the borders of endothelial cells (Muller et al, 1989; Newman et al, 1990). Homophilic interaction of the amino terminal portion (Ig domains 1 and 2) of leukocyte PECAM with the same domains of endothelial cell PECAM is required for diapedesis. Blocking this interaction with a domain-specific monoclonal antibody or with a soluble form of PECAM-1 as a competitive inhibitor blocks diapedesis in vitro (Liao et al, 1995; Muller et al, 1993) and in vivo (Bogen et al, 1994; Liao et al, 1997). Leukocytes blocked at the PECAMdependent step are tightly adherent to the apical surface, but cannot pass pseudopods into the intercellular junction (Liao et al, 1995; Muller et al, 1993). Although blocking PECAM reduces transendothelial migration by up to $90 \%$ in vitro and in vivo (Bogen et al, 1994; Christofidou-Solomidou et al, 1997; Gumina et al, 1996; Liao et al, 1995, 1997, 1999; Muller et al, 1993; Murohara et al, 1996; Vaporciyan et al, 1993), it cannot be the only molecule involved in this particular step. No combination of anti-PECAM reagents ever blocks transendothelial migration completely. Furthermore, mice in which PECAM-1 has been genetically deleted by homologous recombination are able to recruit leukocytes to sites of inflammation in a variety of models, with only minor differences from wild-type animals (Duncan et al, 1999). All known cell adhesion molecules are expressed at wild-type levels in these mice (Duncan et al, 1999). Therefore, it is probable that the "knockout mice" have expanded the function of whichever molecule(s) usually mediate the $10 \%$ to $20 \%$ of diapedesis that is PECAM-independent to levels that can provide a normal inflammatory response. Alternatively, the molecule(s) responsible for PECAM-independent transmigration await discovery.

CD99 is a 32-kd, highly O-glycosylated molecule that is expressed on the surfaces of most leukocytes and was recently appreciated to be concentrated at the borders between confluent endothelial cells (Schenkel et al, 2002). CD99 does not belong to any known protein family. CD99 had been described as a costimulatory molecule on $\mathrm{T}$ cells and thymocytes (Bernard A, 1997; Bernard G et al, 1995, 1997, 2000; Choi et al, 1998; Hahn et al, 1997; Waclaveicek et al, 1998; Wingett et al, 1999), but its role in transmigration was only recently appreciated (Schenkel et al, 2002). Similar to PECAM-1, CD99 functions in a homophilic manner in transmigration, and blockade of CD99 on either leukocytes or endothelial cells blocks diapedesis of monocytes as well as blockade of CD99 on both cell types. Fab fragments of anti-CD99 monoclonal antibody blocked diapedesis by more than $90 \%$ in an in vitro model of transendothelial migration (Schenkel et al, 2002). CD99 controls a step in diapedesis that is different from that controlled by PECAM-1. Blocking both CD99 and PECAM-1 gave an additive effect, essentially abolishing diapedesis completely (Schenkel et al, 2002). Furthermore, CD99 controls a step in diapedesis that is distal to the one controlled by PECAM-1. Monocytes blocked at the CD99dependent step were arrested part-way across the endothelial junction. The leading edge of the monocyte was below the endothelial cell, but a major portion of the cell was still in the junction itself, and the trailing edge remained on the apical surface of the endothelial monolayer (Fig. 2). This demonstrated that diapedesis is controlled by at least two separate sets of homophilic interactions acting at two separate steps. There may well be additional steps in the transmigration process that we are unaware of because we have not yet discovered ways to inhibit them.

It will be important to follow up these in vitro findings on monocytes with studies of the role of CD99 in diapedesis of neutrophils and lymphocytes. The murine homolog of CD99 has not been reported. Once it has been found, it will be critical to determine the role of CD99 in various inflammatory models in vivo.

Two other junctional adhesion molecules, junctional adhesion molecule (JAM-1) and VE-cadherin, components of tight and adherens junctions, respectively, have been reported to play a role in transmigration (Muller, 2001). However, evidence of their roles in diapedesis is controversial. Antibody against murine JAM reduced inflammation in two animal models (Del Maschio et al, 1999; Martin-Padura et al, 1998). However, the same monoclonal antibody failed to block in a similar model of meningitis due to a different organism (Lechner et al, 2000). The human form of JAM-1 has been identified, but in one report monoclonal antibodies against it did not have an effect on leukocyte migration (Liu et al, 2000). A recent paper using a polyclonal antibody reported a role for JAM-1 in transmigration of $\mathrm{T}$ cells and PMN in vitro (Ostermann et al, 2002). The novelty here was that JAM-1 was engaging LFA-1 on the leukocytes in a heterophilic manner that involved the membrane-proximal domain of JAM-1, the immunoglobulin domain not involved in homophilic JAM-JAM adhesion.

Interfering with VE-cadherin by infusing a monoclonal antibody into mice before stimulation of peritonitis was shown to increase the rate at which neutrophils arrived at the site of inflammation (Gotsch et al, 1997). This suggested that integrity of the adherens junction was a barrier to neutrophil migration. On the other hand, in vitro studies performed under flow conditions suggest that VE-cadherin is transiently removed from the junctions during diapedesis (Allport et al, 2000; Shaw et al, 2001). In that case the role played by the antibody is unclear, unless it facilitates this process.

\section{Migration Across Basal Lamina and Through Extracellular Matrix}

After leukocytes traverse the junctions between endothelial cells, they must pass across the subendothelial basal lamina and then through extracellular matrix (ECM) of the interstitial tissues to arrive at their goal. Even less is known at the molecular level about these processes than about transmigration. Migration across basal lamina is clearly controlled by distinct molecular processes, because leukocytes can be arrested after transmigration, but are unable to pass the basement membrane (Duncan et al, 1999; Liao et al, 1995; Wakelin et al, 1996). 


\section{PECAM-1 Homophilic CD99 PECAM-1 Heterophilic}



Figure 2.

Diapedesis is controlled by different molecules acting at different steps. Homophilic interaction between the amino terminal domains of PECAM-1 on the leukocyte and the amino terminal domains of PECAM-1 concentrated at the endothelial borders are important for the initial passage of the leukocyte pseudopod into the intercellular junction. This is inferred from experiments in which these interactions are blocked and the leukocytes remain tightly adherent to the apical surface of the endothelium at the cell borders, unable to insert pseudopods into the junction. Homophilic interaction between CD99 on the leukocyte and CD99 concentrated at the borders of endothelial cells is involved farther along in the leukocyte's passage. Blockade of CD99 function arrests the leukocyte part way through the junction unable to migrate farther. Heterophilic interaction of membrane-proximal immunoglobulin domain 6 of leukocyte PECAM-1 with some unidentified component(s) of the basal lamina is involved in passage of leukocytes across the subendothelial basement membrane. Selective blockade of PECAM domain 6 arrests leukocytes between the basal surface of the endothelial cell and the basal lamina. The site of blockade of diapedesis when junctional adhesion molecule (JAM)- 1 is blocked has not been demonstrated. However, because JAM-1 is believed to be expressed in complexes close to the apical surface of the endothelium, one would predict that leukocytes would be arrested close to the apical surface.

There is a role for PECAM-1 in the passage of leukocytes across basal lamina. This is quite distinct from the role played by PECAM-1 in diapedesis. There is no PECAM in the basal lamina. Diapedesis involves a heterophilic interaction between the membraneproximal domain 6 of leukocyte PECAM and some unknown component(s) of the basal lamina (Liao et al, 1995; Muller et al, 1997). Soluble heparin and heparan sulfate, which are components of the basal lamina, can also block passage across the basal lamina in vitro, presumably by competitive inhibition (Liao et al, 1995). However, PECAM-1 does not bind directly to heparin (Sun et al, 1998). Perhaps heparan sulfate is a coligand, or heparan sulfate polymers present a multivalent ligand to PECAM on the leukocyte. Whatever, the mechanism, a role for PECAM-1 in migration across the basal lamina has also been demonstrated in vivo (Duncan et al, 1999; Wakelin et al, 1996). As in diapedesis, PECAM-1 is clearly not the only molecule that controls transmigration, because PECAMdeficient mice show only a delay in the migration of leukocytes at this step; with time, leukocytes arrive at the inflammatory site in normal numbers (Duncan et al, 1999).

Diapedesis and migration across basal lamina both involve adhesive interactions in three dimensions rather than the two dimensional surface of the endothelial cell. However, the thickness of the endothelial cell and the basal lamina are small compared with the diameter of the leukocyte. Once in the interstitial tissues, the leukocytes locomote within a matrix that is fully three dimensional. This involves interactions that are likely to be very different from the ones modeled in traditional two-dimensional systems (Friedl and Brocker, 2000; Mandeville et al, 1997).

Roles for both $\beta_{1}$ and $\beta_{2}$ integrins in migration through ECM have been demonstrated. Studies in vivo showed that the $\beta_{1}$ integrins of neutrophils, which are up-regulated on transmigration, act in concert with $\beta_{2}$ integrins to promote migration (Werr et al, 1998). This is not surprising considering the known importance of $\beta_{1}$ integrins in binding various ECM proteins. In the study above, $\alpha_{4} \beta_{1}$, and/or $\alpha_{5} \beta_{1}$ were found to be the specific integrins involved. The $\beta_{2}$ integrins, especially $\alpha_{M} \beta_{2}$ have been identified in some systems to promote migration through ECM. In addition to binding ICAM-1 and ICAM-2, this integrin binds to fibrinogen and some of its proteolytic cleavage products known to be components of the ECM, especially at sites of inflammation (Forsyth et al, 2001).

\section{Epilogue}

This brief and necessarily incomplete review of the cellular interactions of the inflammatory response cannot possibly convey all that is known about these interactions or, more important, what must still be learned. The cellular interactions involved in orches- 
trating the inflammatory response are multiple and complex. There is undoubtedly even more complexity involved than we realize in the regulation of these interactions, because inflammation must be turned off as well as on. Furthermore, as alluded to earlier, there are mechanisms to selectively recruit subtypes of leukocytes to particular vascular beds in specific organs. We must gain a more complete understanding of the cellular and molecular interactions between leukocytes and endothelial cells if we are to control this process. Despite the rapid progress in this field, investigation of these critical interactions will keep medical researchers occupied for many years.

\section{Acknowledgements}

I wish to thank Dr. Robert Eddy for preparing the figures.

\section{References}

Allport JR, Ding H, Collins T, Gerritsen ME, and Luscinskas FW (1997). Endothelial-dependent mechanisms regulate leukocyte transmigration: A process involving the proteasome and disruption of the vascular endothelial-cadherin complex and endothelial cell-to-cell junctions. J Exp Med 186:517527.

Allport JR, Muller WA, and Luscinskas FW (2000). Monocytes induce reversible focal changes in vascular endothelial cadherin complex during transendothelial migration under flow. J Cell Biol 148:203-216.

Anderson DC and Springer TA (1987). Leukocyte adhesion deficiency: An inherited defect in the Mac-a, LFA-1, and p150,95 glycoproteins. Ann Rey Med 38:175-194.

Baggiolini M (1998). Chemokines and leukocyte traffic. Nature 392:565-568.

Baggiolini M (2001). Chemokines in pathology and medicine. J Intern Med 250:91-104.

Berlin C, Bargatze RF, Campbell JJ, von Andrian UH, Szabo MC, Hasslen SR, Nelson RD, Berg EL, Erlandsen SL, and Butcher EC (1995). Alpha 4 integrins mediate lymphocyte attachment and rolling under physiologic flow. Cell 80:413422.

Bernard A (1997). CD99 workshop panel report. In: Kishimoto $\mathrm{T}$, editor. Leukocyte Typing VI. Proceedings of the Vlth international leukocyte differentiation antigen workshop, Kobe, Japan, 1996. London: Garland Publishers, 75-77.

Bernard G, Breittmayer J-P, de Matteis M, Trampont $P$, Hofman P, Senik A, and Bernard A (1997). Apoptosis of immature thymocytes mediated by E2/CD99. J Immunol 158:2543-2550.

Bernard G, Raimondi V, Alberti I, Pourtein M, Widjenes J, Ticchioni M, and Bernard A (2000). CD99 (E2) up-regulates $\alpha_{4 \beta 1}$-dependent $\mathrm{T}$ cell adhesion to inflamed vascular endothelium under flow conditions. Eur J Immunol 30:3061-3065.

Bernard G, Zoccola D, Deckert M, Breittmayer J-P, Aussel C, and Bernard A (1995). The E2 molecule (CD99) specifically triggers homotypic aggregation of $\mathrm{CD} 4^{+} \mathrm{CD} 8^{+}$thymocytes. $\mathrm{J}$ Immunol 154:26-32.
Bogen S, Pak J, Garifallou M, Deng X, and Muller WA (1994). Monoclonal antibody to murine PECAM-1 [CD31] blocks acute inflammation in vivo. J Exp Med 179:1059-1064.

Burns AR, Bowden RA, MacDonell SD, Walker DC, Odebunmi TO, Donnachie EM, Simon SI, Entman ML, and Smith CW (2000). Analysis of tight junctions during neutrophil transendothelial migration. J Cell Sci 113:45-57.

Burns AR, Walker DC, Brown ES, Thurmon LT, Bowden RA, Keese CR, Simon SI, Entman ML, and Smith CW (1997). Neutrophil transendothelial migration is independent of tight junctions and occurs preferentially at tricellular corners. $\mathrm{J} \mathrm{Im-}$ munol 159:2893-2903.

Burns AR, Walker DC, and Smith CW (2001). Relationship between tight junctions and leukocyte transmigration. In: Cereijido $\mathrm{M}$ and Anderson J, editors. Tight junctions. Boca Raton: CRC Press, 629-652.

Campbell JJ and Butcher EC (2000). Chemokines in tissuespecific and microenvironment specific lymphocyte homing. Curr Opin Immunol 12:336-341.

Carlos TM and Harlan JM (1994). Leukocyte-endothelial cell adhesion molecules. Blood 84:2068-2101.

Choi EY, Park WS, Jung KC, Kim SH, Kim YY, Lee WJ, and Park SH (1998). Engagement of CD99 induces up-regulation of TCR and MHC class I and II molecules on the surface of human thymocytes. J Immunol 161:749-754.

Christofidou-Solomidou M, Nakada MT, Williams J, Muller WA, and Delisser HM (1997). Neutrophil platelet endothelial cell adhesion molecule-1 participates in neutrophil recruitment at inflammatory sites and is down-regulated after leukocyte extravasation. J Immunol 158:4872-4878.

Cinamon G, Shinder V, and Alon R (2001). Shear forces promote lymphocyte migration across vascular endothelium bearing apical chemokines. Nature Immunol 2:515-522.

Coxon A, Tang T, and Mayadas TN (1999). Cytokineactivated endothelial cells delay neutrophil apoptosis in vitro and in vivo. A role for granulocyte/macrophage colonystimulating factor. J Exp Med 190:923-934.

Cuvelier SL and Patel KD (2001). Shear-dependent eosinophil transmigration on interleukin 4-stimulated endothelial cells: A role for endothelium-associated eotaxin-3. J Exp Med 194:1699-1709.

Dejana E, Corada M, and Lampugnani M (1995). Endothelial cell-to-cell junctions. FASEB J 9:910-918.

Del Maschio A, De Luigi A, Martin-Padura I, Brockhaus M, Bartafai T, Fruscella P, Adorini L, Martino G, Furlan R, De Simoni MG, and Dejana E (1999). Leukocyte recruitment in the cerebrospinal fluid of mice with experimental meningitis is inhibited by an antibody to junctional adhesion molecule (JAM). J Exp Med 190:1351-1356.

Del Maschio A, Zanetti A, Corada M, Rival Y, Ruco L, Lampugnani MG, and Dejana E (1996). Polymorphonuclear leukocyte adhesion triggers the disorganization of endothelial cell-to-cell adherens junctions. J Cell Biol 135:497-510.

Duncan GS, Andrew DP, Takimoto H, Kaufman SA, Yoshida H, Spellberg J, de la Pompa JL, Elia A, Wakeham A, KaranTamir B, Muller WA, Sendali G, Zukowski MM, and Mak TW (1999). Genetic evidence for functional redundancy of platelet/endothelial cell adhesion molecule-1 (PECAM-1): CD31deficient mice reveal PECAM-1-dependent and PECAM-1independent functions. J Immunol 162:3022-3030. 
Etzioni A, Doerschuk CM, and Harlan JM (1999). Of man and mouse: Leukocyte and endothelial adhesion molecule deficiencies. Blood 94:3281-3288.

Feng D, Nagy JA, Pyne K, Dvorak HF, and Dvorak AM (1998). Neutrophils emigrate from venules by a transendothelial cell pathway in response to fMLP. J Exp Med 187:903-915.

Forsyth CB, Solovjov DA, Ugarova TP, and Plow EF (2001). Integrin $\alpha_{\mathrm{M} \beta 2}$-mediated cell migration to fibrinogen and its recognition peptides. J Exp Med 193:1123-1133.

Friedl P and Brocker E-B (2000). The biology of cell locomotion within three-dimensional extracellular matrix. Cell Mol Life Sci 57:41-64.

Gautam N, Herwald H, Hedqvist P, and Lindbom L (2000). Signaling via $\beta_{2}$ integrins triggers neutrophil-dependent alteration in endothelial barrier function. J Exp Med 191:18291839.

Gautam N, Olofsson AM, Herwald H, Iversen LF, LundgrenAkerlund $\mathrm{E}$, Hedqvist $\mathrm{P}$, Arfors K-E, Flodgaard H, and Lindbom L (2001). Heparin-binding protein (HBP/CAP37): A missing link in neutrophil-evoked alteration of vascular permeability. Nat Med 7:1123-1127.

Gopalan PK, Burns AR, Simon SI, Sparks S, McIntyre LV, and Smith CW (2000). Preferential sites for stationary adhesion of neutrophils to cytokine-stimulated HUVEC under flow conditions. J Leukoc Biol 68:47-57.

Gotsch U, Borges E, Bosse R, Boggemeyer E, Simon M, Mossmann H, and Vestweber D (1997). VE-cadherin antibody accelerates neutrophil recruitment in vivo. J Cell Sci 110: 583-588.

Gumina RJ, Schultz JE, Yao Z, Kenny D, Warltier DC, Newman PJ, and Gross GJ (1996). Antibody to platelet/ endothelial cell adhesion molecule-1 reduces myocardial infarct size in a rat model of ischemia-reperfusion injury. Circulation 94:3327-3333.

Hahn J-H, Kim MK, Choi EY, Kim SH, Sohn HW, Ham DI, Chung DH, Kim TJ, Lee WJ, Park CK, Ree HJ, and Park SH (1997). CD99 (MIC2) regulates the LFA-1/ICAM-1-mediated adhesion of lymphocytes, and its gene encodes both positive and negative regulators of cellular adhesion. J Immunol 159:2250-2258.

Hajjar KA, Esmon NL, Marcus AJ, and Muller WA (2001). Vascular function in hemostasis, 6th ed. In: Beutler E, Coller BS, Lichtman MA, Kipps TJ, and Seligsohn U, editors. Vascular function in hemostasis, 6th ed. New York: McGrawHill Medical Publishing Division, 1451-1469.

Henderson RB, Lim LHK, Tessier PA, Gavins FNE, Mathies M, Perretti M, and Hogg N (2001). The use of lymphocyte function-associated antigen (LFA)-1-deficient mice to determine the role of LFA-1, Mac-1, and $\alpha 4$ integrin in the inflammatory response of neutrophils. J Exp Med 194:219226.

Hixenbaugh EA, Goeckeler ZM, Papaiya NN, Wysolmerski RB, Silverstein SC, and Huang AJ (1997). Chemoattractantstimulated neutrophils induce regulatory myosin light chain phosphorylation and isometric tension development in endothelial cells. Am J Physiol 273:H981-H988.

Huang AJ, Furie MB, Nicholson SC, Fischbarg J, Liebovitch LS, and Silverstein SC (1988). Effects of human neutrophil chemotaxis across human endothelial cell monolayers on the permeability of these monolayers to ions and macromolecules. J Cell Physiol 135:355-366.
Huang AJ, Manning JE, Bandak TM, Ratau MC, Hanser KR, and Silverstein SC (1993). Endothelial cell cytosolic free calcium regulates neutrophil migration across monolayers of endothelial cells. J Cell Biol 120:1371-1380.

Hynes RO (1992). Integrins: Versatility, modulation, and signaling in cell adhesion. Cell 69:11-25.

Issekutz TB, Miyasaka M, and Issekutz AC (1996). Rat blood neutrophils express very late antigen 4 and it mediates migration to arthritic joint and dermal inflammation. J Exp Med 183:2175-2184.

Kaplanski G, Fabrigoule M, Boulay V, Dinarello CA, Bongrand P, Kaplanski S, and Farnarier C (1997). Thrombin induces endothelial type II activation in vitro: IL-1 and TNF-alphaindependent IL-8 secretion and E-selectin expression. J Immunol 158:5435-5441.

Kaplanski G, Farnarier C, Kaplanski S, Porat R, Shapiro L, Bongrand P, and Dinarello CA (1994). Interleukin-1 induces interleukin- 8 secretion from endothelial cells by a juxtacrine mechanism. Blood 84:4242-4248.

Kitayama J, Hidemura A, Saito H, and Nagawa H (2000). Shear stress affects migration behavior of polymorphonuclear cells arrested on endothelium. Cell Immunol 203:3946.

Kubes P and Kanwar S (1994). Histamine induces leukocyte rolling in post-capillary venules. A P-selectin-mediated event. $\mathrm{J}$ Immunol 152:3570-3577.

Lawson MA and Maxfield FR (1995). Ca2+- and calcineurindependent recycling of an integrin to the front of migrating neutrophils. Nature 377:75-79.

Lechner F, Sahrbacher U, Suter T, Frei K, Brockhaus M, Koedel U, and Fontana A (2000). Antibodies to the junctional adhesion molecule cause disruption of endothelial cells and do not prevent leukocye influx into the meninges after viral or bacterial infection. J Infect Dis 182:978-982.

Liao F, Ali J, Greene T, and Muller WA (1997). Soluble domain 1 of platelet-endothelial cell adhesion molecule (PECAM) is sufficient to block transendothelial migration in vitro and in vivo. J Exp Med 185:1349-1357.

Liao F, Huynh HK, Eiroa A, Greene T, Polizzi E, and Muller WA (1995). Migration of monocytes across endothelium and passage through extracellular matrix involve separate molecular domains of PECAM-1. J Exp Med 182:1337-1343.

Liao F, Schenkel AR, and Muller WA (1999). Transgenic mice expressing different levels of soluble platelet/endothelial cell adhesion molecule-IgG display distinct inflammatory phenotypes. J Immunol 163:5640-5648.

Liu Y, Nusrat A, Schnell FJ, Reaves TA, Walsh S, Pochet M, and Parkos CA (2000). Human junction adhesion molecule regulates tight junction resealing in epithelia. J Cell Sci 113:2363-2374.

Lorenzon P, Vecile E, Nardon E, Ferrero E, Harlan JM, Tedesco F, and Dobrina A (1998). Endothelial cell E-and $\mathrm{P}$-selectin and vascular cell adhesion molecule-1 function as signaling receptors. J Cell Biol 142:1381-1391.

Mandeville JTH, Lawson MA, and Maxfield FR (1997). Dynamic imaging of neutrophil migration in three dimensions: Mechanical interactions between cells and matrix. J Leuk Biol 61:188-200.

Marchesi VT (1961). The site of leukocyte emigration during inflammation. Q J Exp Physiol Cogn Med Sci 46:115-118. 
Marchesi VT and Florey HW (1960). Electron micrographic observations on the emigration of leukocytes. Q J Exp Physiol Cogn Med Sci 45:343-347.

Martin-Padura I, Lostaglio S, Schneemann M, Williams L, Romano M, Fruscella P, Panzeri C, Stoppacciaro A, Ruco L, Villa A, Simmons D, and Dejana E (1998). Junctional adhesion molecule, a novel member of the immunoglobulin superfamily that distributes at intercellular junctions and modulates monocyte transmigration. J Cell Biol 142:117-127.

Middleton J, Neil S, Wintle J, Clark-Lewis I, Moore H, Lam C, Auer M, Hub E, and Rot A (1997). Transcytosis and surface presentation of IL-8 by venular endothelial cells. Cell 91:385395.

Miller J, Knorr R, Ferrone M, Houdei R, Carron CP, and Dustin ML (1995). Intercellular adhesion molecule-1 dimerization and its consequences for adhesion mediated by lymphocyte function associated molecule-1. J Exp Med 182:1231-1241.

Moll TE, Dejana E, and Vestweber D (1998). In vitro degradation of endothelial catenins by a neutrophil protease. J Cell Biol 140:403-407.

Muller WA (1999). Leukocyte-endothelial cell adhesion molecules in transendothelial migration, 3rd ed. In: Gallin JI and Snyderman R, editors. Inflammation: Basic principles and clinical correlates. Philadelphia: Lippincott Williams \& Wilkins, 585-592.

Muller WA (2001). Migration of leukocytes across endothelial junctions: Some concepts and controversies. Microc 8:181193.

Muller WA, Greene T, and Liao F (1997). Transendothelial migration and interstitial migration of monocytes are mediated by separate domains of monocyte CD31. In: Kishimoto $\mathrm{T}$, editor. Leukocyte typing VI. Proceedings of the Vlth international leukocyte differentiation antigen/workshop, Kobe, Japan, 1996. London: Garland Publishers, 370-372.

Muller WA, Ratti CM, McDonnell SL, and Cohn ZA (1989). A human endothelial cell-restricted, externally disposed plasmalemmal protein enriched in intercellular junctions. J Exp Med 170:399-414.

Muller WA and Weigl S (1992). Monocyte-selective transendothelial migration: Dissection of the binding and transmigration phases by an in vitro assay. J Exp Med 176:819-828.

Muller WA, Weigl SA, Deng X, and Phillips DM (1993). PECAM-1 is required for transendothelial migration of leukocytes. J Exp Med 178:449-460.

Murohara T, Delyani JA, Albelda SM, and Lefer AM (1996). Blockade of platelet endothelial cell adhesion molecule-1 protects against myocardial ischemia and reperfusion injury in cats. J Immunol 156:3550-3557.

Newman PJ, Berndt MC, Gorski J, White II GC, Lyman S, Paddock C, and Muller WA (1990). PECAM-1 [CD31] cloning and relation to adhesion molecules of the immunoglobulin gene superfamily. Science 247:1219-1222.

Ostermann G, Weber KSC, Zernecke A, Schroder A, and Weber C (2002). JAM-1 is a ligand of the $\beta_{2}$ integrin LFA-1 involved in transendothelial migration of leukocytes. Nature Immunol 3:151-158.

Pober JS and Cotran RS (1990). The role of endothelial cells in inflammation. Transplantation 50:537-544.
Pan J, Xia L, Yao L, and McEver RP (1998). Tumor necrosis factor-alpha- or lipopolysaccharide-induced expression of the murine P-selectin gene in endothelial cells involves novel kappaB sites and a variant activating transcription factor/ cAMP response element. J Biol Chem 273:10067-10077.

Pouyani T and Seed B (1995). PSGL-1 recognition of $\mathrm{P}$-selectin is controlled by a tyrosine sulfation consensus at the PSGL-1 amino terminus. Cell 83:333-343.

Randolph GJ, Beaulieu S, Lebecque S, Steinman RM, and Muller WA (1998). Differentiation of monocytes into dendritic cells in a model of transendothelial migration. Science 282: $480-483$.

Randolph GJ, Inaba K, Robbiani DF, Steinman RM, and Muller WA (1999). Differentiation of phagocytic monocytes into lymph node dendritic cells in vivo. Immunity 11:753-761.

Reilly PL, Woska JRJ, Jeanfavre DD, McNally E, Rothlein R, and Bormann BJ (1995). The native structure of intercellular adhesion molecule-1 (ICAM-1) is a dimer. Correlation with binding to LFA-1. J Immunol 155:529-532.

Saito $H$, Minamiya $Y$, Kitamura $M$, Saito $S$, Enomoto $K$, Terada K, and Ogawa J (1998). Endothelial myosin light chain kinase regulates neutrophil migration across human umbilical vein endothelial cell monolayer. J Immunol 161:1533-1540.

Sako D, Comess KM, Barone KM, Camphausen RT, Cumming DA, and Shaw GD (1995). A sulfated peptide segment at the amino terminus of PSGL-1 is critical for P-selectin binding. Cell 83:323-331.

Schenkel AR, Mamdouh Z, Chen X, Liebman RM, and Muller WA (2002). CD99 plays a major role in the migration of monocytes through endothelial junctions. Nature Immunol 3:143-150

Shaw SK, Bamba PS, Perkins BN, and Luscinskas FW (2001). Real-time imaging of vascular endothelial-cadherin during transmigration across endothelium. J Immunol 167: 2323-2330.

Simon SI, Burns AR, Taylor AD, Gopalan PK, Lynam EB, Sklar LA, and Smith CW (1995). L-selectin (CD62L) crosslinking signals neutrophil adhesive functions via the Mac-1 (CD11b/CD18) $\beta_{2}$ integrin. J Immunol 155:1502-1514.

Smith CW, Rothlein R, Hüghes BJ, Mariscalso MM, Rudloff HE, Schmalstieg FC, and Anderson DC (1988). Recognition of an endothelial determinant for CD18-dependent human neutrophil adherence and transendothelial migration. J Clin Invest 82:1746-1756.

Su W-H, Chen H, Huang J, and Jen CJ (2000). Endothelial $\left[\mathrm{Ca}^{2+}\right]_{\mathrm{i}}$ signaling during transmigration of polymorphonuclear leukocytes. Blood 96:3816-3822.

Sun Q-H, Paddock C, Visentin GP, Zukowski MM, Muller WA, and Newman PJ (1998). Cell surface glycosaminoglycans do not serve as ligands for PECAM-1. PECAM-1 is not a heparin-binding protein. J Biol Chem 273:11483-11490.

Tanaka Y, Adams DH, Hubscher S, Hirano H, Siebenlist U, and Shaw $S$ (1993). T-cell adhesion induced by proteoglycan-immunobilized cytokine MIP-1 beta. Nature 361:79-82.

Vaporciyan AA, Delisser HM, Yan H-C, Mendiguren II, Thom SR, Jones ML, Ward PA, and Albelda SM (1993). Involvement of platelet-endothelial cell adhesion molecule- 1 in neutrophil recruitment in vivo. Science 262:1580-1582. 
Vestweber D and Blanks JE (1999). Mechanisms that regulate the function of the selectins and their ligands. Physiol Rev 79:181-213.

von Adrian UH, Hasslen SR, Nelson RD, Erlandsen SL, and Butcher E (1995). A central role for microvillous receptor presentation in leukocyte adhesion under flow. Cell 82:989999.

Waclaveicek M, Majdic O, Stulnig T, Berger M, SunderPlassmann R, Zlabinger GJ, Baumruker T, Stockel J, Ebner C, Knapp W, and Pickl WF (1998). CD99 engagement on human peripheral blood $T$ cells results in TCR/CD3dependent cellular activation and allows for Th1-restricted cytokine production. J Immunol 161:4671-4678.

Wakelin MW, Sanz M-J, Dewar A, Albelda SM, Larkin SW, Boughton-Smith N, Williams TJ, and Nourshargh S (1996). An anti-platelet/endothelial cell adhesion molecule- 1 antibody inhibits leukocyte extravasation from mesenteric microvessels in vivo by blocking the passage through basement membrane. J Exp Med 184:229-239.

Werr J, Xie X, Hedqvist P, Ruoslahti E, and Lindbom L (1998). $\beta_{1}$ integrins are critically involved in neutrophil locomotion in extravascular tissue in vivo. J Exp Med 187:2091-2096.
Wingett D, Forcier K, and Nielson CP (1999). A role for CD99 in T cell activation. Cell Immunol 193:17-23.

Worthylake RA, Lemoine S, Watson JM, and Burridge $\mathrm{K}$ (2001). RhoA is required for monocyte tail retraction during transendothelial migration. J Cell Biol 154:147-160.

Yao L, Pan J, Setiadi H, Patel KD, and McEver RP (1996). Interleukin 4 or oncostatin $\mathrm{M}$ induces a prolonged increase in P-selectin mRNA and protein in human endothelial cells. $J$ Exp Med 184:81-92.

Zimmerman GA, Mclntyre TM, Mehra M, and Prescott SM (1990). Endothelial cell-associated platelet-activating factor: a novel mechanism for signaling inercellular adhesion. J Cell Biol 110:529-540.

Zlotnick A and Yoshie O (2000). Chemokines: A new classification system and their role in immunity. Immunity 12:121127.

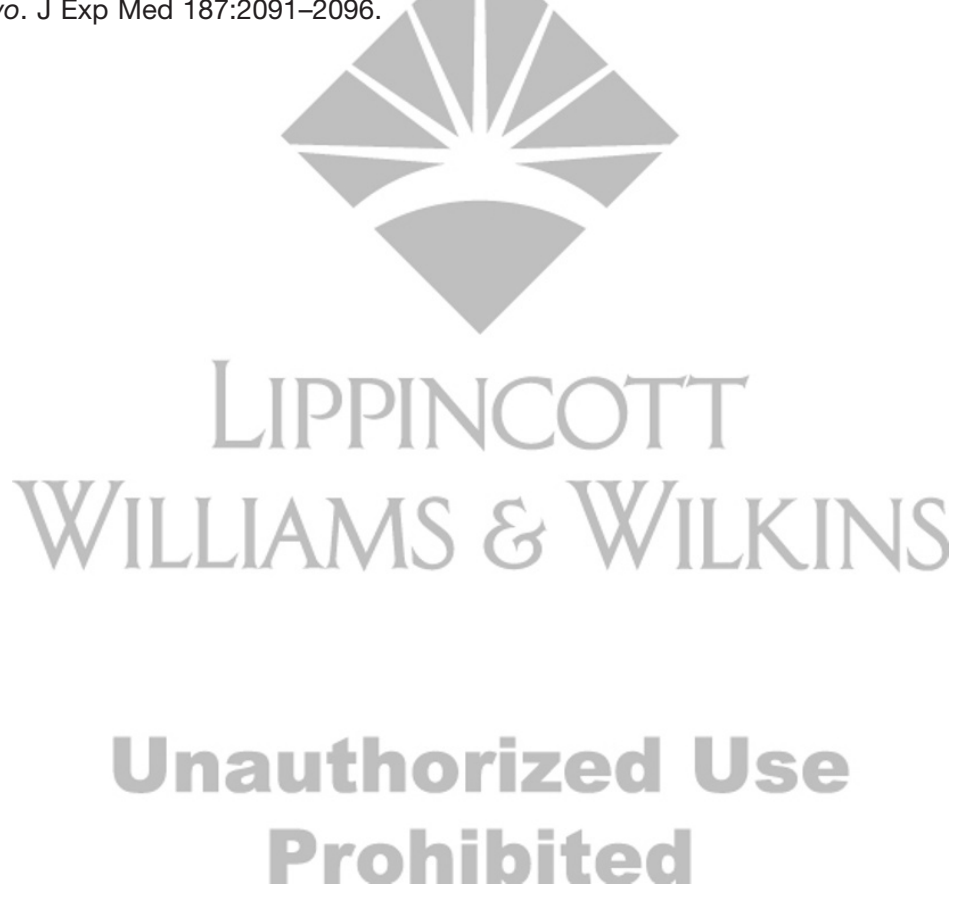

